\title{
Multibranch entrainment and slow evolution among branches in coupled oscillators
}

\author{
Toru Aonishi ${ }^{1}$ and Masato Okada ${ }^{2,1}$ \\ ${ }^{1}$ Brain Science Institute, RIKEN, 2-1 Hirosawa, \\ Wako-shi, Saitama, 351-0198, Japan \\ ${ }^{2}$ ERATO Kawato Dynamic Brain Project, 2-2 Hikaridai, \\ Seika- cho, Soraku-gun, Kyoto 619-0288, Japan
}

(Dated: November 23, 2018)

\begin{abstract}
In globally coupled oscillators, it is believed that strong higher harmonics of coupling functions are essential for multibranch entrainment (MBE), in which there exist many stable states, whose number scales as $\sim O(\exp N)$ (where $N$ is the system size). The existence of MBE implies the non-ergodicity of the system. Then, because this apparent breaking of ergodicity is caused by microscopic energy barriers, this seems to be in conflict with a basic principle of statistical physics. In this paper, using macroscopic dynamical theories, we demonstrate that there is no such ergodicity breaking, and such a system slowly evolves among branch states, jumping over microscopic energy barriers due to the influence of thermal noise. This phenomenon can be regarded as an example of slow dynamics driven by a perturbation along a neutrally stable manifold consisting of an infinite number of branch states.

PACS numbers: 05.45.Xt, 75.10.Nr, 87.18.Sn
\end{abstract}


An Ising model with a ferromagnetic interaction has a simple phase space, and below some critical temperature it only possesses two macroscopic states, two oppositely directed ferromagnetic states which have the same energy. Contrastingly, in the case of ferromagnetic coupled oscillators with coupling functions containing strong higher harmonics, each oscillator is multi-stable, and as a result, the configuration observed in a simulation will consist of individual clusters characterized by different phases. Daido showed through numerical simulations that there exist many stable states, whose number scales as $\sim O(\exp N)$ (where $N$ is the system size) [1]. This complex mutual entrainment is called multibranch entrainment (MBE). This phenomenon share the same mechanism of so-called clustering, which has been found in various systems, e.g., globally coupled maps [2] and pulse-coupled oscillators [3]. When such systems exhibit periodic behavior, their dynamics can be described by phase equations with coupling functions containing strong higher harmonics [3].

It is a general principle of statistical mechanics that ergodicity can be broken only by macroscopic barriers of the free energy whose heights scale as $\sim O(N)$. Here, we evaluate the free energy of a system of phase oscillators with coupling functions containing strong higher harmonics. Then, we demonstrate that MBE means ergodicity breaking caused by microscopic free energy barriers whose heights scale as $\sim O(1)$. As this result reveals, MBE seems to be in conflict with a basic principle of statistical physics, because a system can jump over these microscopic barriers due to the influence of thermal noise. Next, we apply the moment approach [曰, 5] to such a system and study properties of its relaxation process. Then we elucidate the effect of microscopic energy barriers and demonstrate thermodynamical instability of MBE.

The ferromagnetic Ising model and the ferromagnetic analog network, governed by a Markovian process and a continuous process, are in the general class of time-dependent Ginzburg-Landau (TDGL) models [6], because in such systems, the dynamics of macroscopic order parameters are described by the steepest-descent of the free energy. A ferromagnetic coupled oscillator, however, is not in the class of TDGL models. In this paper, we construct a Markovian system that has the same free energy as a ferromagnetic coupled oscillator but, nonetheless, belongs to the class of TDGL models. Then, comparing this TDGL system and non-TDGL ferromagnetic coupled oscillator system, we make clear that microscopic energy barriers have no effect on the relaxation of the system in TDGL models we consider but that they cause the relaxation to become slow in non-TDGL models we consider. Our results 
suggest that such slowing of relaxation is characteristic only of non-TDGL models.

We propose the following phase model with uniform all-to-all coupling by modifying Daido's model [1]:

$$
\begin{aligned}
\frac{d \phi_{i}}{d t}=\omega_{i} & -\frac{1}{N} \sum_{j=1}^{N}\left[(1-a) \sin \left(\phi_{i}-\phi_{j}\right)\right. \\
& \left.+a \sin \left(2\left(\phi_{i}-\phi_{j}\right)\right)\right]+n_{i}(t) .
\end{aligned}
$$

Here $\phi_{i}$ is the phase of the $i$ th oscillator (with a total of $N$ ), and $\omega_{i}$ represents its quenched natural frequency. The natural frequencies are randomly distributed with a density represented by $g(\omega)=\left(2 \pi \sigma^{2}\right)^{-1 / 2} \exp \left(-\omega^{2} / 2 \sigma^{2}\right)$. The parameter $a$ denotes the degree of mixture of the fundamental harmonic component and the higher harmonic component in the coupling functions $(0 \leq a \leq 1)$. This model belongs to the class of ferromagnetic systems and does not exhibit frustration in the mutual interaction. The function $n_{i}$ represents a Gaussian independent white-noise process satisfying $\left\langle n_{i}(t)\right\rangle=0,\left\langle n_{i}(t) n_{j}\left(t^{\prime}\right)\right\rangle=2 T \delta_{i j} \delta\left(t-t^{\prime}\right)$, where $T$ is the temperature of the system, and the inverse temperature is defined as $\beta=1 / T$. Daido used a coupling of the form $\sin x+a \cos 2 x$ instead of that in Eq. (1). In the case of Daido's model, when a gradually increase, another stable solution suddenly appears apart form original stable solution in coupling function. This sudden appearance of another stable solution also occurs in our model. Thus, the bifurcation structure of Daido's model is qualitatively equal to that of our model. Moreover, when there is no frequency disorder, detailed balance holds in our model. Therefore, we can analyze the MBE of our model in a transparent manner.

For convenience, we define $s_{i}=\exp \left(i \phi_{i}\right)$ and rewrite Eq. (1) in the following form with bare mean fields:

$$
\begin{aligned}
\frac{d \phi_{i}}{d t}=\omega_{i} & +\frac{1}{2 i}\left[(1-a)\left(\bar{s}_{i} m_{1}-s_{i} \overline{m_{1}}\right)\right. \\
& \left.+a\left(\bar{s}_{i}^{2} m_{2}-s_{i}^{2} \overline{m_{2}}\right)\right]+n_{i}(t) .
\end{aligned}
$$

Here the overline denotes the complex conjugate. The quantity $m_{1}$ is a mean field consisting of the fundamental harmonics $\left\{s_{j}\right\}_{j=1, \cdots, N}$, and $m_{2}$ is a mean field consisting of the higher harmonics $\left\{s_{j}^{2}\right\}_{j=1, \cdots, N}: m_{1}=\frac{1}{N} \sum_{j}^{N} s_{j} ; m_{2}=\frac{1}{N} \sum_{j}^{N} s_{j}^{2}$. 
Equation (2) obeys the Fokker-Planck equation

$$
\begin{aligned}
& \frac{\partial p(\phi \mid \omega, t)}{\partial t}=T \frac{\partial^{2} p(\phi \mid \omega, t)}{\partial \phi^{2}}-\frac{\partial}{\partial \phi}(v(\phi, \omega) p(\phi \mid \omega, t)) \\
& v(\phi, \omega)=\omega \\
& \quad+\frac{1}{2 i}\left((1-a)\left(\bar{s} m_{1}-s \overline{m_{1}}\right)+a\left(\bar{s}^{2} m_{2}-s^{2} \overline{m_{2}}\right)\right)
\end{aligned}
$$

where $p(\phi \mid \omega, t)$ denotes the one-oscillator probability density at time $t$. The site index $i$ of $\omega$ and $\phi$ can be omitted, because Eq. (2) has already been reduced to a one-body system.

We derive the macroscopic dynamics of the system by using the moment approach [4, 5]. We define the moments of $p(\phi \mid \omega, t)$ as

$$
\lambda_{n}(\omega, t)=\int_{0}^{2 \pi} d \phi p(\phi \mid \omega, t) \exp (-i n \phi) .
$$

The motion of these moments obeys the simple equation

$$
\begin{aligned}
& \frac{d \lambda_{n}(\omega, t)}{d t}=-T n^{2} \lambda_{n}(\omega, t)+i n \omega \lambda_{n}(\omega, t) \\
& -\frac{n(1-a)}{2}\left(m_{1} \lambda_{n+1}(\omega, t)-\overline{m_{1}} \lambda_{n-1}(\omega, t)\right) \\
& -\frac{a n}{2}\left(m_{2} \lambda_{n+2}(\omega, t)-\overline{m_{2}} \lambda_{n-2}(\omega, t)\right),
\end{aligned}
$$

which is derived through a moment expansion of Eq. (3). Equation (6) possesses a hierarchal structure, because the $n$th moment depends on neighboring moments [the $(n-2)$ th, $(n-1)$ th, $n$ th, $(n+1)$ th and $(n+2)$ th moments]. The 0 th moment is found trivially to be $\lambda_{0}(\omega, t)=1$. The mean fields $m_{1}$ and $m_{2}$ are given by $\overline{m_{1}}=\int d \omega g(\omega) \lambda_{1}(\omega, t), \quad \overline{m_{2}}=\int d \omega g(\omega) \lambda_{2}(\omega, t)$. When there is no frequency disorder, we obtain $\overline{m_{1}}=\lambda_{1}(0, t)$ and $\overline{m_{2}}=\lambda_{2}(0, t)$. If $T \neq 0$, higher moments rapidly decay, since the coefficient of the damping factor in Eq. (6) is $T n^{2}$. Therefore, even if higher moments are omitted in the numerical calculation of Eq. (6), highly accurate results can be obtained. For the convenience of later discussions, we call the system without the thermal noise term the "Liouville part", because Eq. (6) without the diffusion part (i.e., the damping factor) is equivalent to the so-called Liouville equation. The diffusion part in Eq. (6) can be regarded as a perturbation of the Liouville equation.

When there is no frequency disorder [i.e. when $g(\omega)=\delta(\omega)$ ], detailed balance holds, since the coupling function is an odd function [5, 7]. Thus, in the limit $\sigma \rightarrow 0$, this model can be mapped to the equilibrium system. In this case, there exists a free energy, which enables us to apply equilibrium statistical mechanical methods to the system. Then we can 
obtain the Gibbs distribution and partition function

$$
P(\phi)=e^{-\beta H(\phi)} / Z, \quad Z=\int_{0}^{2 \pi} d \phi e^{-\beta H(\phi)},
$$

in equilibrium [5], where $H$ denotes the Hamiltonian expressed by

$$
\begin{aligned}
& H(\boldsymbol{\phi})= \\
& -\sum_{i}\left(\frac{1-a}{2}\left(\bar{s}_{i} m_{1}+s_{i} \overline{m_{1}}\right)+\frac{a}{4}\left(\bar{s}_{i}^{2} m_{2}+s_{i}^{2} \overline{m_{2}}\right)\right) .
\end{aligned}
$$

We thus obtain the free energy $f=-1 /(\beta N) \log Z$. Using the saddle-point method to evaluate the integral in $Z$, for large $N$, we derive the free energy as

$$
\begin{gathered}
f\left(m_{1}, m_{2}, \overline{m_{1}}, \overline{m_{2}}\right)=\frac{1-a}{2}\left|m_{1}\right|^{2}+\frac{a}{4}\left|m_{2}\right|^{2} \\
-\frac{1}{\beta} \log \left\langle e^{\beta\left(\frac{1-a}{2}\left(\bar{s} m_{1}+s \overline{m_{1}}\right)+\frac{a}{4}\left(\bar{s}^{2} m_{2}+s^{2} \overline{m_{2}}\right)\right)}\right\rangle_{\phi} .
\end{gathered}
$$

In the limit $T \rightarrow 0$, this becomes

$$
f\left(m_{1}, m_{2}, \overline{m_{1}}, \overline{m_{2}}\right)=\frac{1-a}{2}\left|m_{1}\right|^{2}+\frac{a}{4}\left|m_{2}\right|^{2}=\frac{H}{N} .
$$

This is the Hamiltonian normalized with respect to $N$.

Let us consider the fixed points of Eq. (2) in the limit $T \rightarrow 0$ and $\sigma \rightarrow 0$. Figure 1 displays a typical example of a fundamental harmonic component and a higher harmonic component of Eq. (2) in the case $\frac{d \phi_{i}}{d t}=0$. Namely, figure 1 shows two curves $h_{1}\left(\phi_{i}\right)=$ $-\frac{1-a}{2 i}\left(\bar{s}_{i} m_{1}-s_{i} \overline{m_{1}}\right)$ and $h_{2}\left(\phi_{i}\right)=\frac{a}{2 i}\left(\bar{s}_{i}^{2} m_{2}-s_{i}^{2} \overline{m_{2}}\right)$. If $a$ is sufficiently large, Eq. (包) admits four solutions corresponding to intersection points of the fundamental harmonic component and the higher harmonic component, as shown in Fig. 11. The filled circles and open circles correspond to stable solutions and unstable solutions, respectively. We thus find that this system is microscopically bistable. The two enclosed areas $A_{1}$ and $A_{2}$ in Fig. 1 are, respectively, equal to the energies of the two stable solutions, as measured with respect to the unstable solution between them. The microscopic energy barrier discussed in introduction is the energy barrier between these stable solutions. It is a principle of statistical physics that in a situation like this, only the stable solution with the larger enclosed area can be realized, because only the stable solution with the lowest energy is thermodynamically stable. This rule is referred to as Maxwell's rule [8]. Note that two stable solutions have the same energy only if $a=1$. However, if system is non-ergodic, multiple states can coexist, and Maxwell's 
rule does not hold. In this case, depending on the initial conditions, each oscillator comes to belong to one of two clusters. Then the total number of stable solutions in the whole system is $O\left(2^{N}\right) \sim O(\exp N)$. This is the situation involving MBE.

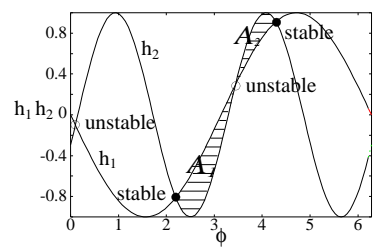

FIG. 1: Sketch of microscopic bistability.
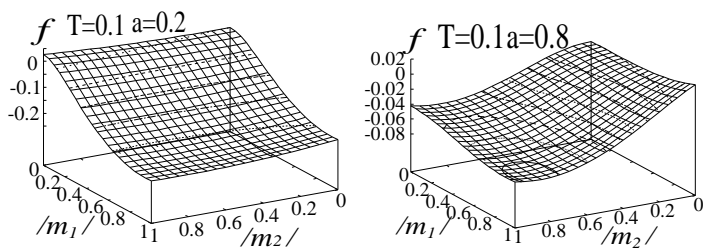

FIG. 2: Macroscopic landscape of the free energy.

Here, we demonstrate that microscopic multi-valley of the free energy is not reflected in the macroscopic structure of the free energy. Figure $\mathbf{Q}$ displays the macroscopic landscape of the free energy (Eq. (9)) as a function of $\left|m_{1}\right|$ and $\left|m_{2}\right|$. The left figure corresponds to the case of a monostable system $(a=0.2)$, and the right figure corresponds to the case of a bistable system $(a=0.8)$. There are no any local minima of the two energy surfaces. Thus, it is seen even in the case of a bistable system, the free energy does not possess a multi-valley structure macroscopically. From Eq. (10), it is clear that, even in the limit $T \rightarrow 0$, there is no macroscopic multi-valley structure in the free energy. Note that in the limit $T \rightarrow 0$, the critical value of $a$, representing the boundary between monostability and bistability, is given by $a_{c}=0.467$.

As the macroscopic structure of the free energy reveals, it seems that the ergodicity breaking by microscopic barriers does not occur. However, when $g(\omega)=\delta(\omega), T \rightarrow 0$ and $a>a_{c}, \lambda_{n}(0, \infty)=r+(1-r)(-1)^{n}$ corresponding to some branch state is a stable solution of Eq. (6). In this case, the mean fields $m_{1}$ and $m_{2}$ are given by $m_{1}=2 r-1$ and $m_{2}=1$. These moments yield the probability density function $p(\phi \mid 0, \infty)=r \delta(\phi)+(1-r) \delta(\phi-\pi)$, where $r$ denotes the ratio of oscillators at $\phi=0$ to those at $\phi=\pi$. This solution is satisfied 
for any $r(0 \leq r \leq 1)$. In this case, these solutions form a line attractor parameterized by $r$ : $M=\left\{r+(1-r)(-1)^{n} \mid 0 \leq r \leq 1\right\}$. Within this attractor, all of the solutions are neutrally stable. We thus find that in the limit $N \rightarrow \infty$, the set of solutions forming MBE can be parameterized by the single variable $r$.

Therefore, in the limit $T \rightarrow 0$, the global ergodicity demonstrated by the macroscopic structure of the free energy seems to be in conflict with the existence of $M$ revealed by the moment approach. In the following numerical calculations, we evaluate the dynamical properties of this system at low temperature, and then answer the question what happens in the limit $T \rightarrow 0$. In the numerical simulations we now discuss, the initial conditions of the system were assigned as random numbers with the density function $p(\phi \mid \omega, 0)=$ $q \delta(\phi)+(1-q) \delta(\phi-\pi)$, where $q$ denotes the ratio of oscillators at $\phi=0$ to those at $\phi=\pi$. Thus, initial conditions were chosen on the manifold $M$.
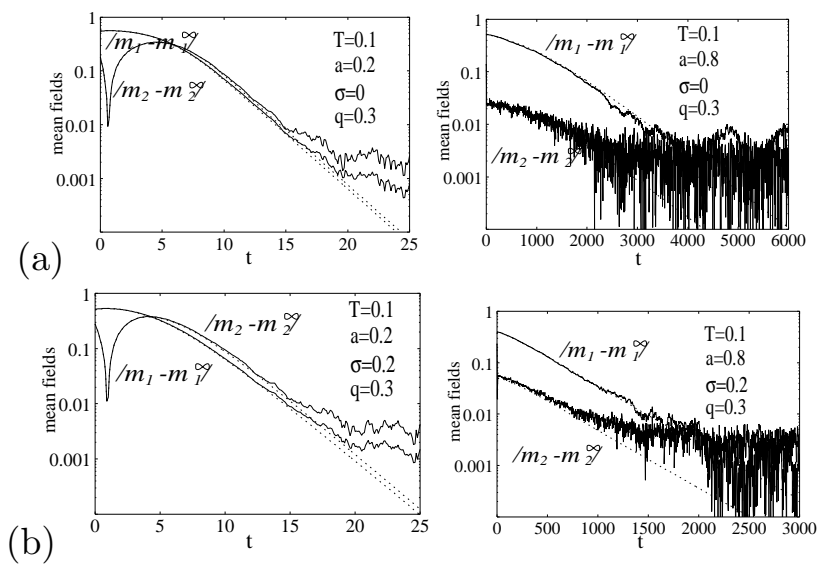

FIG. 3: Temporal behavior of $m_{1}-m_{1}^{\infty}$ and $m_{2}-m_{2}^{\infty}$ : (a) $\sigma=0$. (b) $\sigma=0.2$. The dashed curves were obtained analytically, while the solid curves were obtained from numerical simulations.

Figures 3(a) displays the temporal behavior of $\left|m_{1}-m_{1}^{\infty}\right|$ and $\left|m_{2}-m_{2}^{\infty}\right|$, where $m_{1}^{\infty}$ and $m_{2}^{\infty}$ denote values of $m_{1}$ and $m_{2}$ in an equilibrium state corresponding to the minimum point of the free energy shown in Fig. 2. The dashed curves in these figures are the analytical results obtained from Eq. (6), and the solid curves are numerical results. As these figures reveal, the analytical results fits in with the simulation results.

We now calculate the time constant $\tau$ of the system using logarithm plots. According to Fig. 3(a), the relaxation process to equilibrium states is characterized by the exponential convergence. The time constant of this convergence, $\tau$, for the bistable system $(a=0.8)$ is 
about 100 times larger than that for the monostable system $(a=0.2)$. We also evaluate the time constant $\tau$ as a function of $T$ in the case of a bistable system $(a=0.8)$. As Fig. 5 (a) reveals, $\tau$ diverges to $\infty$ in the limit $T \rightarrow 0$.

Note that in the initial stages of the relaxation process, when initial states are far from $M$, the speed of convergence for the system with $a=0.8$ is almost the same as that for the system with $a=0.2$. During the approach to the equilibrium state, the relaxation process suddenly switches from a process of fast dynamics to a process of slow dynamics (see Fig. 田). The mechanism responsible for this switch is the following. Since the thermal perturbation (the diffusion part in Eq. (6) ) is very small, for solutions far from $M$, the perturbation is negligible, and they are attracted rapidly to $M$, just as in the unperturbed system. (This rapid attraction is called the "fast dynamics".) Now note that the Liouville part vanishes on $M$. For this reason, once solutions enter the neighborhood of $M$, where the effects of the Liouville part and the perturbation become comparable, the solution begins to move slowly along $M$ toward the ground state. (This is called the "slow dynamics".)

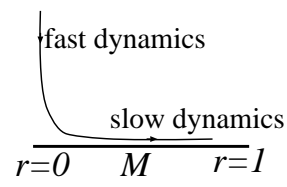

FIG. 4: Sketch of dynamical flow around $M$.

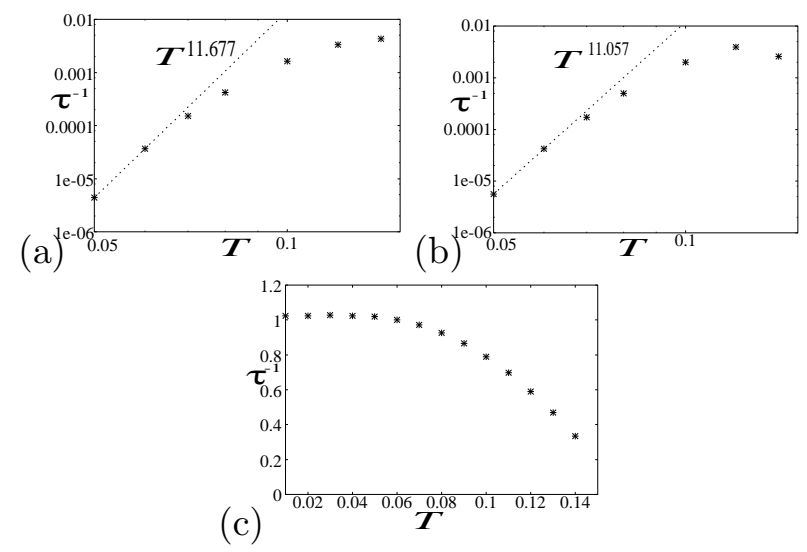

FIG. 5: Time constant $\tau$ as a function of $T$ : (a) $\sigma=0, a=0.8, q=0.3$; (b) $\sigma=0.1, a=0.8, q=0.3$;

(c) Markovian system $(\sigma=0, a=0.8, q=0.3)$.

In order to compare the non-TDGL system considered above to a TDGL system, we evaluated the time constant $\tau$ for a Glauber system. This system was constructed to have 
the same Gibbs distribution as Eq. (7) in the equilibrium state. Thus, its macroscopic dynamics are described by the steepest-descent of the free energy given in Eq. (9). As Fig. 5(c) reveals, in this case $\tau$ converges to some finite value as $T \rightarrow 0$, even for a bistable system ( $a=0.8$ ). In the limit $T \rightarrow 0$, the Glauber system chooses only the stable solution with the lower energy. We thus find that microscopic energy barriers do not affect the relaxation process of the Glauber system. This contrasts with what we have found for the relaxation process of the continuous system Eq. (11), which is hampered by the microscopic energy barriers, resulting in a slow (infinitely slow in the $T \rightarrow 0$ limit) relaxation to the ground state.

In order to study the effect of the frequency disorder, we also measured the temporal behavior of $\left|m_{1}-m_{1}^{\infty}\right|$ and $\left|m_{2}-m_{2}^{\infty}\right|$ in the case $\sigma \neq 0$ (see Fig. 3(b).) Here, $m_{1}^{\infty}$ and $m_{2}^{\infty}$ denote values of $m_{1}$ and $m_{2}$ in a macroscopic steady state [1]. Even if there is frequency disorder, the time constant of this convergence, $\tau$, for the bistable system $(a=0.8)$ is about 100 times larger than that for the monostable system $(a=0.2)$. As shown in Fig. 5(b), $\tau$ diverges to $\infty$ in the limit $T \rightarrow 0$.

In conclusion, the system we have considered evolves slowly through a series of branch states to the ground state, jumping over microscopic energy barriers through the influence of thermal noise. The time constant $\tau$ characterizing this relaxation process diverges to $\infty$ in the limit $T \rightarrow 0$. From the macroscopic viewpoint, this phenomenon can be regarded as slow dynamics driven by a weak thermal perturbation along the neutrally stabile manifold $M$ consisting of an infinite number of branch states. From these results, we can conclude that, in the long term, MBE is unstable thermodynamically, but in the short term, the system preserves phase patterns highly correlated with the initial conditions, because its relaxation time is very long.

There exist some theoretical studies of coupled oscillators based on the assumption that the effect of the frequency disorder is equivalent to that of thermal noise [9, 10]. According to the present results, we can conclude that the effect of quenched frequency disorder is far different from that of thermal noise [11, 12].

[1] H. Daido, Phys. Rev. Let. 77, 1406 (1996).

[2] K. Kaneko, Physca D 41, 137 (1990). 
[3] U. Ernst, K. Pawelzik, and T. Geisel, Phys. Rev. Let. 74, 1570 (1995).

[4] C. J. Pèrez and F. Ritort, J. Phys. A 30, 8095 (1997).

[5] L. L. Bonilla, C. J. Pèrez, F. Ritort, and J. Soler, Phys. Rev. Let. 81, 3643 (1998).

[6] P. C. Hohenberg and B. I. Halperin, Rev. Mod. Phys. 49, 435 (1977).

[7] T. Aonishi, Phys. Rev. E 58, 4865 (1998).

[8] M. Shiino and T. Fukai, Phys. Rev. E 48, 867 (1993).

[9] A. Arenas and C. J. P. Vicente, Europhys. Lett. 26, 79 (1994).

[10] K. Park and M. Y. Choi, Phys. Rev. E 52, 2907 (1995).

[11] Y. Braiman, H. G. E. Hentschel, F. Family, C. Mak, and J. Krim, Phys. Rev. E 59, R4737 (1999).

[12] H. G. E. Hentschel, F. Family, and Y. Braiman, Phys. Rev. Let. 83, 104 (1999). 